
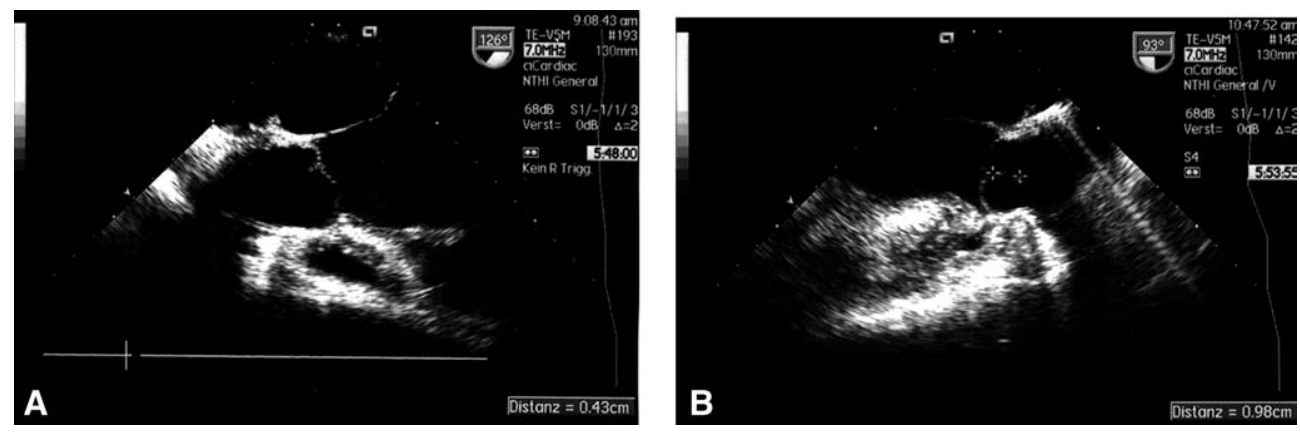

Figure 2. A, Preoperative transesophageal echocardiogram of a patient who presented with grade III aortic regurgitation after valve reimplantation in a different institution. There is some prolapse of the fused cusp and an effective height of $4 \mathrm{~mm}$. Both findings were confirmed by intraoperative measurements. B, Postoperative transesophageal echocardiogram of the same patient after shortening of both cusps. The valve is competent, and an effective height of $10 \mathrm{~mm}$ has been achieved.

\title{
Endovascular treatment of concomitant patent ductus arteriosus and type $B$ aortic dissection in a patient with pulmonary artery dissection
}

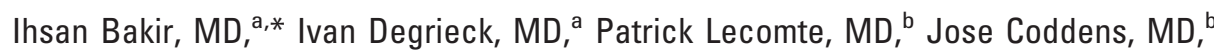
Luc Foubert MD, PhD, ${ }^{\text {b }}$ Alex Heyse, MD, ${ }^{c}$ and Hugo Vanermen, MD, ${ }^{a}$ Aalst, Belgium

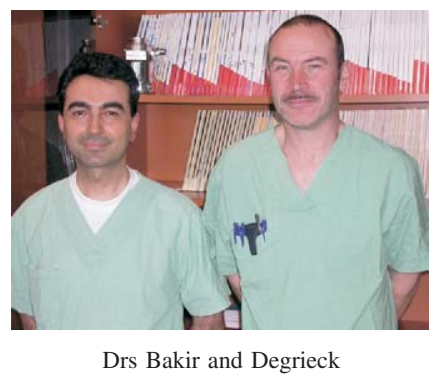

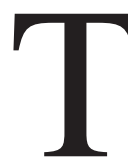

he ideal management of patent ductus arteriosus (PDA) in the older patent is still controversial. Increasing evidence in the literature ${ }^{1,2}$ indicates that transcatheter closure of PDA in this age group is safe and effective. A case of a patient with symptomatic PDA accompanying a chronic type B aortic dissection and silent pulmonary artery (PA) dissection is described.

\section{Clinical Summary}

A 74-year-old woman was admitted with diffuse peripheral edema and orthopnea. Physical examination revealed a continuous murmur on the precordial region. Assessment with transesophageal echocar-

From the Departments of Thoracic and Cardiovascular Surgery ${ }^{\mathrm{a}}$ and Anesthesiology and Critical Care Medicine, ${ }^{\mathrm{b}}$ OLV Clinic, Aalst, Belgium, and the Department of Cardiology, AZ ZVB, ${ }^{\mathrm{c}}$ Ronse, Belgium.

*Dr. Bakir is affiliated with the Department of Cardiovascular Surgery, Siyami Ersek Cardiovascular and Thoracic Surgery Center, Haydarpasa, Istanbul, Turkey. Received for publication Feb 21, 2006; accepted for publication April 10, 2006.

Address for reprints: Ihsan Bakir, MD, OLV Clinic, Cardiovascular and Thoracic Surgery Department, Moorselbaan 164, 9300 Aalst, Belgium (E-mail: ihsanbak@yahoo.com).

J Thorac Cardiovasc Surg 2006;132:438-40

$0022-5223 / \$ 32.00$

Copyright (C) 2006 by The American Association for Thoracic Surgery doi:10.1016/j.jtcvs.2006.04.013 diography revealed an ascending aortic aneurysm of $4.6 \mathrm{~cm}$, dilated right ventricle, dissection of the descending aorta, and an open ductus Botalli. Pulmonary hypertension was estimated from pressure measurements. Computed tomographic (CT) scan confirmed the diagnosis of PDA (diameter $10 \mathrm{~mm}$ ) and type B aortic dissection (Figure 1, $A$ and $B$ ). CT scan also demonstrated a dissection in the truncus pulmonalis (Figure 1, $B$ and $C$ ). It was shown that the flow in the PDA originated from the false lumen of the dissected descending aorta (Figure 1, A) and terminated in the dissected main PA (Figure $1, B$ and $C$ ).

To reduce the risks associated with surgery and age-related comorbidities, the decision was made to close the PDA and concomitantly treat the type B aortic dissection with a stent-graft. With the patient under general anesthesia, the right femoral artery was surgically isolated and a $22 \mathrm{~F}$ introducer sheath was inserted thorough the right femoral artery. An endovascular stent-graft (Gore TAG $40 \mathrm{~mm}$; W. L. Gore \& Associates, Inc, Newark, Del) was inserted into the descending thoracic aorta under fluoroscopic guidance. Aortography confirmed that the stent-graft was properly positioned and that both the origin of the aortic dissection and the PDA were adequately closed (Figure 2, $A$ and $B$ ). The postoperative course was uneventful, and the patient's dyspnea and peripheral edema were diminished after the procedure. A CT scan after 1 week demonstrated satisfactory graft position, without endoleak, and complete closure of the PDA. At 3-month follow-up there were no clinical signs of heart failure: no dyspnea, no peripheral edema, and no pleural effusions. The continuous systolic-diastolic murmur of PDA was no longer observed. 


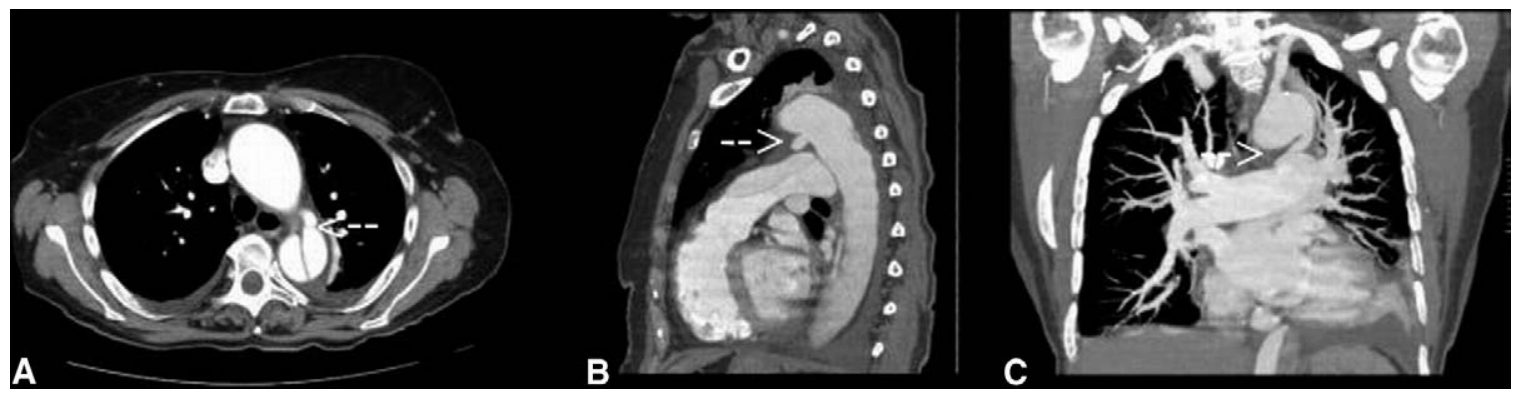

Figure 1. A, Axial view of CT scan indicating PDA (arrow) and chronic type B aortic dissection. PDA is originating from false lumen of dissected aorta. B, Sagittal view of preoperative CT scan demonstrating PDA (arrow) and PA dissection. C, Frontal view of preoperative CT scan demonstrating PDA (arrow) and PA dissection.

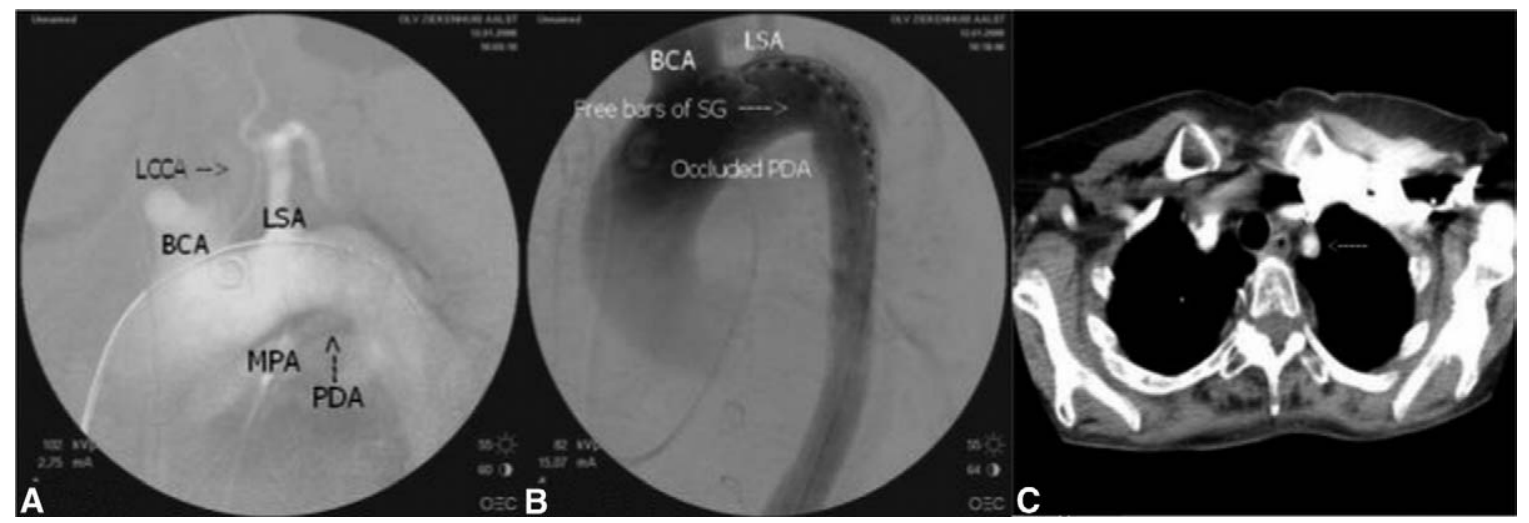

Figure 2. A, Preoperative control arteriography indicating PDA before stent deployment. LCCA, Left common carotid artery; $L S A$, left subclavian artery; $B C A$, brachiocephalic artery; MPA, main PA. B, Immediate postprocedural arteriography shows stent-graft covering entry of PDA except left subclavian artery (LSA). Shunt has disappeared. BCA, Brachiocephalic artery; SG, stent-graft. C, Postprocedural 3-month follow-up CT image indicates thrombosing left subclavian artery (arrow) in its origin.

Echocardiographic examination revealed a PA pressure of $36 \mathrm{~mm} \mathrm{Hg}$, in contrast to the perioperative PA pressure measurement of $49 \mathrm{~mm}$ $\mathrm{Hg}$. Late CT images also demonstrated ideal graft positioning without endoleak. The only extra finding was the thrombosing left subclavian artery in its origin (Figure 2, C). Within the 3-month period, PA dissection had not progressed relative to that seen on previous images.

\section{Discussion}

PDA occlusion is indicated in adults to prevent the hemodynamic consequences of left-to-right shunts and to protect against endocarditis. ${ }^{3}$ Although surgical closure remains the accepted treatment option, the risk of surgery is high in the older patient because of the fragility of neighboring calcified aorta, which may lead to surgical difficulties necessitating cardiopulmonary bypass and graft replacement of the proximal descending thoracic aorta. ${ }^{2,3}$ Longstanding left-to-right shunt is also associated with pulmonary hypertension or chronic ventricular dysfunction. ${ }^{3}$ On the other hand, persistent pulmonary hypertension may cause dissection in the PA, which may lead to pulmonary hemorrhage or sudden death. ${ }^{4}$ Our patient is one of the few survivors without serious rupture or bleeding complicating this rare triple pathologic entity.

Transcatheter closure of the PDA has become an attractive alternative to surgery in adults. ${ }^{1-3}$ The most common approach is multiple coil implantation. ${ }^{2}$ However, coil closure frequently allows residual shunts in large PDAs (diameter $>5 \mathrm{~mm}$ ). The new generation of devices can be applied to PDAs that are moderate to large $(\leq 11 \mathrm{~mm}){ }^{2}$ In our case, concomitant finding of type B aortic dissection ruled out this type of treatment. Stent-grafting can be applied regardless of the size of the PDA. According to an extensive PubMed search, this exclusion technique had never been used electively for concomitant treatment of type B aortic dissection and closure of PDA in a patient with accompanying PA dissection. Closure of the inflow of the PDA and treatment of the type B aortic dissection at the same time appeared to us simple and safe. 
The drawback of the procedure is the possible occlusion of the left subclavian artery. Surgical transposition of the left subclavian artery before the stent-graft placement is the traditional option. ${ }^{2}$ Several articles have recently demonstrated the safety of the intentional coverage of the left subclavian artery without prophylactic surgical transposition, although subclavian steal syndrome developed in some patients, necessitating revascularization of the left subclavian artery during follow-up. ${ }^{5}$

We report a case of successful closure of a large PDA and concomitant treatment of chronic type B aortic dissection with the simple and safe endovascular stent-grafting technique. Stent-grafting may be the first-line treatment option for closing large PDAs in the older patient, especially when chronic type B dissection is also present. Occlusion of the PDA also may protect against further overflow or dissection, and possible rupture of the PA.

\section{References}

1. Ozmen J, Granger EK, Robinson D, White GH, Wilson M. Operation for adult patent ductus arteriosus using an aortic stent-graft technique. Heart Lung Circ. 2005;14:54-7.

2. Saito N, Kimura T, Toma M, Sasaki K, Kita T, Imura M, et al. Transcatheter closure of patent ductus arteriosus with Inoue singlebranched stent graft. J Thorac Cardiovasc Surg. 2005;130:1203-4.

3. Rogues F, Hennequin JL, Sanchez B, Ridarch A, Rousseau H. Aortic stent-graft for patent ductus arteriosus in adults: the aortic exclusion technique. Ann Thorac Surg. 2001;71:1708-9.

4. Ejima K, Uchida T, Hen Y, Nishio Y, Nomoto F, Uchida Y, et al. Silent pulmonary artery dissection in a patient with Eisenmenger syndrome due to ventricular septal defect: a case report. J Cardiol. 2005;46:33-7.

5. Rehders TC, Petzsch M, Ince H, Kische S, Korber T, Koschyk DH, et al. Intentional occlusion of the left subclavian artery during stentgraft implantation in the thoracic aorta: risk and relevance. $J$ Endovasc Ther. 2004;11:659-66. 